\title{
Robot Football Team from Minho University
}

\author{
Carlos Machado, Ilídio Costa, Sérgio Sampaio, Fernando Ribeiro \\ Grupo de Automação e Robótica, Industrial Electronics Department, University of Minho \\ Campus de Azurém, 4000 Guimarães, PORTUGAL \\ fernando@dei.uminho.pt \\ http://www.robotica.del.umınho.pt/
}

\begin{abstract}
This paper describes an Autonomous Mobile Robot team which plays football, developed by the Group of Automation and Robotics at the Industrial Electronics department of the University of Minho, in Guimarães (Portugal). In this competition each team is free to use and/or build all the different electronics, sensory systems, playing algorithms, etc. as far as they cope with the rules imposed by the organisation. Instead of using several different sensors increasing electronics complexity, this team decided to use only one major sensor: a vision system with a small colour camera. All the image processing algorithms were developed from scratch and they consist on the heart of the whole project. This vision system uses an innovative approach: in order to see the whole field, a convex mirror was placed at the top of the robot looking downwards with the video camera looking upwards towards the mirror. This way, the robot can see all around itself with a top view, which means continuous vision of the ball, goals and other robots.
\end{abstract}

\section{Introduction}

Autonomous mobile robots are ever increasing their number of different applications, even in ludic applications or in sports. In the last few years, several robotic football competitions have been organised with participating teams from all over the world. The University of Minho (Portugal) decided to accept the challenge of participating in this competition and found it to be a quite interesting experience and a lot was learned. The final classification of this team at RoboCup'99 was not as good as expected mainly due to problems that have nothing to do with the RoboCup'99 competition. The robots arrived at Stockholm only after the competition had started; equipment had been damaged in the transport; the postponing of some games forced this team to play 4 games in one day with the robots not completely assembled. Besides all these problems, some other teams did not take into consideration the collision avoidance rule, crashing and destroying some of our robots. Our robots were properly programmed to avoid collisions but not to run away from other dangerous robots. However, participating was an extremely good experience, and this team expects to participate again next year hopefully without all these problems.

M. Veloso, E. Pagello, and H. Kitano (Eds.): RoboCup-99, LNAI 1856, pp. 731-734, 2000.

Springer-Verlag Berlin Heidelberg 2000 


\section{Robots}

The robot base used was one from another competition and is made up of very light wood. It consists of a two levels platform with the two wheel voids. On the bottom level was placed the DC/DC converter (between the two wheels) and the two $12 \mathrm{~V}$ 7Ah batteries (one at the front side and the other at the rear side). At the top level, it was placed the computer mother board and respective boards (video and graphics boards). The hardware consisted on a personal computer mother board with a Pentium processor running at $200 \mathrm{MHz}$ (MMX), with 32 Mbytes of memory (although the DOS operating system was used and therefore only 1 Mbyte was used). The hard disk had 2 Gbytes. A colour video camera is used with a frame grabber type Bt848. The communication hardware and software was not ready in time for the RoboCup99 competition and therefore each robot played on his own.

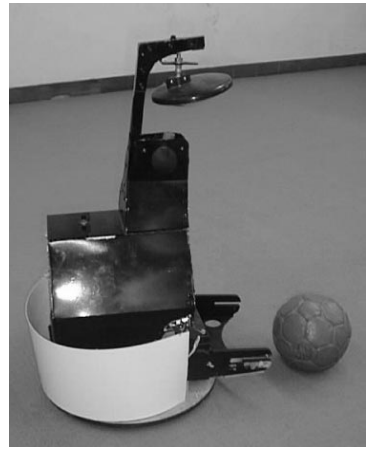

Fig. 1. Photograph of a team's Robot

\section{World Model}

At any given time, these robots are aware of the ball (knowing its direction), both goals direction and their own approximate position on the field. Robots on the field are also seen but not distinguished whether their belong to own or opponent team. This information is kept in the form of a direction variable, and when required they move towards it, updating that direction variable at every frame captured. These robots do not memorise anything else.

\section{Perception}

The only sensor to perceive all the items needed to play a game, is a simple colour camera with its frame grabber plugged on a computer slot. To perceive all those items, these robots grab one image every $20 \mathrm{~ms}$, and the software finds the peak of a certain colour (after removing noise). For example, to track the ball, the software 
searches a peak of red. Since colours depend very much on the light conditions, a calibration is made prior to a game, in order to inform the software what is the minimum value for a red to be a ball. The software tracks down the following items: the ball (by its red colour); the two goals (also by their yellow and blue colours); all other robots (mainly black coloured); the field surrounding walls (mainly white coloured).

These robots avoid collisions, by perceiving as uncollidable items all the black and/or white items. This is the way they avoid walls (mainly white) and other robots (mainly black). The white lines on the green field are ignored because what the robot sees is not "mainly white" due to the slim thickness of the lines.

\section{Communication}

It was this team intention to implement communication, but due to lack of time this was not ready in time for the competition. However, the system is hereby described. It consists of a radio frequency module containing an emitter and a receiver, plugged in each robot, plus one more module in a remote computer allowing to "see" what is going on, on the field, through a graphical animation.

Each of these modules are able to send a message to a certain robot of to all robots. The message is not longer than 255 bytes and contains instructions about actual information (a position, or a decision) or what to do next, and these messages have different levels of importance. Each robot can communicate with one other particular robot or with all of them at the same time. Being so, a complete confusion could be generated and therefore different levels of importance are used. This level depends on the owner position, or distance to the ball, goals, opponent robots, etc. The robots communicate only when it is needed (not all the time) in order to keep the radio environment free for urgent messages to pass through.

\section{Strategy}

In order to drive the ball, these robots use an arch with a re-entrance of $7 \mathrm{~cm}$ (allowed by the rules). This way, ball control is achieved just by pushing it, although a sudden change of direction might mean loosing the ball. These sudden changes of direction are avoided by the robot's software by following longer and wider trajectories.

These robots intercept the ball very easy. When they see the ball, they just go towards it, avoiding collisions with the opponent robot, but insisting and never giving up, until the opponent robot looses the ball. Once they have the ball, they move towards the opponent goal dribbling the opponents (and avoiding collisions). In case they loose the ball, instantaneously start the procedure "following ball" again.

When owning the ball near the opponent goal, these robots do not kick the ball. They run into the goal pushing the ball with their body. This means a disadvantage since most goalies are very good and attack the ball sufficiently fast to avoid scoring. 
This team's goalie is different from the other players only what concerns the direction of the wheels. These are rotated 90 degrees in order to be fast defending the goal rather than moving towards the front. The goalie software is very simple and consists of looking and observing the ball all the time. That is possible with that convex mirror. It then moves sideways in order to keep its body always in the ball's direction no matter how distant this is. When the ball approaches, the goalie kicks the ball with its arch rotating its body, doing a movement like a tennis player with its racket. This movement is very beautiful and improves the quality of the game. This technique not only avoids a goal but also kicks the ball far away from its goal.

Since these robots always have an eye on the ball, their reaction is very simple and efficient. When they don't have the ball, they go towards it and don't give up until they get the ball. Once they have the ball they go towards the opponent goal in order to score, and avoiding obstacles. If, for some rare reason they don't see the ball, they start moving in a spiral until they see the ball (avoiding the walls, of course).

\section{Special Team Features}

The image processing was the most important aspect of this team. It proved to be very consistent, fast and original. All the video drivers were re-written in assembly language in order to take the most out of the video board. This way, 50 frames per second were achieved making the rest of the control program an easy task. All the image processing routines were written in assembly language in order to increase speed and it proved to be very necessary. The general control program and strategy were written in $\mathrm{C}$ language since it did not need to be extremely fast. Sometimes, in two consecutive cycles the same image was analysed (giving the same result as the previous cycle), proving that it was not necessary to have a so fast processor. The mirror technique also proved to be very efficient since everything can be seen at all time. With everything on sight, it is much easier to make the flux control program.

\section{Conclusions}

As main conclusions it can be said that the image processing developed and used by this team is the most important characteristic. It is very reliable, consistent, fast.

The robot movements are very smooth and acceptably unpredictable unlike a typical algorithm with known steps used by many teams.

All the hardware and software of the robots was designed, developed and built by graduate students at the University of Minho. Only four robots were built due to lack of budget. These 4 robots were designed, built, programmed and tested by three industrial electronics students only, plus the team leader making a team of 4 .

Even though this team had had many problems during its participation on RoboCup, the team learned a lot and gained experience. The rules are now clear, new ideas came up by looking at other teams playing, and new improvements will be implemented. This team intends to participate next year, after improving the robots. 